
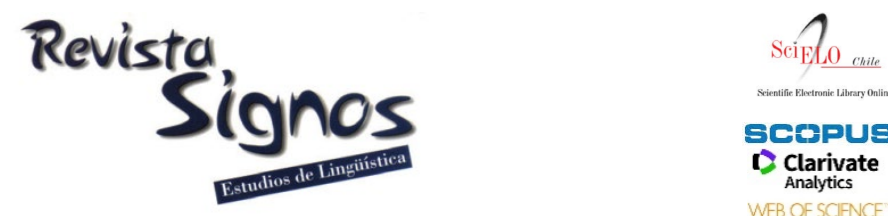

\title{
La habilidad léxico-semántica en la Enfermedad de Alzheimer: Un estudio de la fluidez verbal con categorías semánticas
}

\author{
Lexical-semantic ability in Alzheimer' disease: A study of \\ verbal fluency with semantic categories
}

\section{Olga Ivanova}

UNIVERSIDAD DE SALAMANCA

ESPAÑA

olga.ivanova@usal.es

\section{Israel Martínez-Nicolás}

UNIVERSIDAD DE SALAMANCA

ESPAÑA

israelmani@usal.es

\author{
Juan José García Meilán \\ UNIVERSIDAD DE SALAMANCA \\ ESPAÑA \\ meilan@usal.es
}

Thide E. Llorente

UNIVERSIDAD DE SALAMANCA

ESPAÑA

llorentel@usal.es

Recibido: $10-\mathrm{I}-2019$ / Aceptado: $14-\mathrm{VIII}-2019$
DOI: $10.4067 /$ S0718-09342020000100319

\section{Resumen}

Las primeras alteraciones lingüísticas propias de la Enfermedad de Alzheimer (EA) ocurren en el nivel léxico-semántico, fuertemente dependiente del estado de la memoria del hablante. Los cambios cualitativos en la memoria semántica, derivados de los procesos neurodegenerativos, dan lugar a la aparición de la anomia: la incapacidad de la persona afectada de acceder y recuperar tanto las unidades léxicas como sus bases conceptuales. En este trabajo, nos proponemos estudiar las características cognitivas y semánticas de la afectación anómica en EA e indagar en la posibilidad de su uso en pruebas para la detección temprana de la demencia. Para ello, analizamos los resultados de un modelo experimental de la clásica prueba de fluidez verbal semántica (FVSem), que introduce la división de la misma en cuatro intervalos de 15 segundos con el fin de determinar qué procesos de acceso semántico -los automáticos o los controladosestán alterados en diferentes estados cognitivos de la vejez (hablantes sin patología, hablantes con deterioro cognitivo leve y hablantes con EA). Los resultados de nuestro experimento apuntan a que existe una clara correlación entre el estado cognitivo del hablante y su habilidad léxico-semántica. Asimismo, evidencian, por un lado, que es importante aplicar la prueba de FVSem con duración no inferior a 60 segundos y con distribución en 4 intervalos de 15 segundos, y, por otro lado, que determinadas categorías semánticas -colores y frutas- son más susceptibles a la retrogénesis cognitiva propia de EA.

Palabras Clave: Habilidad léxico-semántica, anomia, redes semánticas, deterioro cognitivo. 


\begin{abstract}
Language impairments in Alzheimer's disease (AD) primarily affect lexical and semantic levels, which significantly depend on the speaker's memory state. Qualitative shifts in semantic memory are due to neurodegenerative processes underlying dementia and give rise to anomia, or the speaker's inability to access and retrieve lexical units and their conceptual backgrounds. In this paper we aim at studying cognitive and semantic features of anomic deficit in $\mathrm{AD}$ and exploring the possibility to apply them in tests for early detection of dementia. For that purpose, we analyze the results from our experimental version of a classical test on semantic verbal fluency (SemVF) with nonpathological aged persons, persons with Mild Cognitive Impairment and persons with AD. The experimental version of the test introduces a division into four 15-minute intervals, in order to find out which processes of semantic access, either automatic or controlled, are impaired in different cognitive states of the elderly. Our results show a correlation between the cognitive state and the lexical-semantic ability of the speaker. Furthermore, they highlight, on the one hand, that the duration of the SemVF test should not be less than 60 seconds, with internal division into 415 -second intervals, and, on the other hand, that some semantic categories -colours and fruit in particularare more prone to be affected by cognitive retrogenesis, which caracterizes AD dementia.
\end{abstract}

Key Words: Lexical-semantic ability, anomia, semantic networks, cognitive impairment.

\title{
INTRODUCCIÓN
}

Una de las características más manifiestas de la Enfermedad de Alzheimer (EA) es el déficit anómico, o la incapacidad de los hablantes para encontrar y utilizar palabras adecuadas tanto en la expresión espontánea como en la denominación de objetos (Cuetos, 2003). Entre los científicos no existe acuerdo sobre la etiología de la anomia en Alzheimer, pero parece más razonable sostener el origen de la misma en la degradación de la memoria semántica. La memoria semántica consiste en la capacidad del hablante de acceder y recuperar diferentes signos lingüísticos, como unidades léxicas (palabras), símbolos verbales, significados y referentes (Palacio \& Toro, 2018), y, básicamente, constituye el diccionario mental compuesto por información léxica, conceptual, de hechos y conocimientos del mundo, necesaria para la producción del lenguaje (Becker \& Overman, 2002). Ya en el estado inicial de la Enfermedad de Alzheimer, los hablantes empiezan a presentar dificultades a la hora de recuperar y transmitir su información personal (sobre todo, datos biográficos), describir escenas y nombrar objetos e imágenes (Jódar Vicente, Barroso Ribal, Brun i Gasca, Dorado Mesa, García Jiménez, Martín Plasencia \& Nieto Barco, 2005). Estas alteraciones tienen una clara dependencia de tales variables como la familiaridad de la unidad conceptual y la edad de adquisición de la unidad léxica y, junto con el aumento de la dificultad de producción verbal con la mayor implicación de la activación semántica, apuntan a que el déficit anómico en EA también es de tipo semántico (Cuetos, 2003). 
A propósito de la anomia en EA, sin embargo, aun quedan muchas interrogantes por contestar y una de las más relevantes al día de hoy es cómo caracterizar y distinguir la anomia vinculada a la demencia de la anomia que caracteriza otras condiciones de senectud, en particular, el envejecimiento no patológico y el deterioro cognitivo leve. La anomia, o la dificultad para nombrar verbalmente un sujeto o un objeto, es un fenómeno muy común en el envejecimiento, que llega a afectar no solo a los hablantes con trastornos del lenguaje sino también a hablantes mayores sanos (Kempler \& Zelinski, 1994). No obstante, la dificultad ocasional de encontrar la palabra buscada, que se debe al gran tamaño del lexicón mental y la velocidad de recuperación de las unidades léxicas específicas (Laine \& Martin, 2006), se diferencia en gran medida de los déficits anómicos derivados de alteraciones en el sistema nervioso central de los humanos. Como síntoma de disfunción lingüística más habitual (Laine \& Martin, 2006), la anomia es un rasgo común a casi todas las afasias y, además, a muchas enfermedades neurodegenerativas (como la demencia semántica, la demencia frontotemporal o la demencia vascular), entre las que destaca la Enfermedad de Alzheimer.

A pesar de su alta incidencia en numerosas condiciones patológicas, la anomia cuenta con un especial peso diagnóstico en EA. En los últimos años, se ha sugerido que la anomia propia de esta demencia sigue unos patrones específicos que permitirían utilizarla como un marcador temprano del desarrollo de EA (Arboleda Ramírez, 2016). Sin embargo, desde la investigación actual se sigue insistiendo en la necesidad de comprender mejor cuáles son los tipos de errores de denominación que caracterizan EA de forma específica (Lima Silagi, Ferreira Bertolucci \& Zazo Ortiz, 2015) y cómo se pueden detectar de forma temprana. Con el fin de disminuir la brecha existente en este respecto, el presente estudio se ha propuesto analizar las manifestaciones prácticas de la anomia asociada a EA y compararla con las manifestaciones anómicas en personas mayores sin patología y en personas mayores con el deterioro cognitivo leve (DCL), un tipo de envejecimiento que manifiesta un deterioro cognitivo más profundo que la norma de deterioro asociado a la vejez y que puede evolucionar hacia la demencia tipo Alzheimer en entre la mitad y los tres cuartos de los casos (Viloria Jiménez, Gil Gregorio \& Yubero Pancorbo, 2009). En este sentido, nuestro objetivo específico ha sido examinar cuantitativamente los procesos de denominación en los tres grupos de estudio a través de la tarea de fluidez verbal semántica, aplicada según diferentes intervalos de tiempo. En el diseño del estudio, hemos partido de la hipótesis de que los hablantes con la Enfermedad de Alzheimer presentarán, frente a los hablantes mayores sin patología y hablantes con DCL, un deterioro significativo de los procesos de denominación en todos los intervalos de tiempo. Ello supone la aceptación de que en EA se encuentran alterados todos los procesos de acceso léxico-semántico, tanto del tipo automatizado como del tipo dependiente del control ejecutivo. En este sentido, es predecible que la capacidad de denominación verbal vaya deteriorándose de forma progresiva con el declive 
cognitivo y ejecutivo que pasa desde la senectud no patológica hasta el DCL y EA, permitiendo desarrollar pruebas adecuadas para la detección temprana de la demencia mediante evaluación de la capacidad de denominación. Este artículo, cuyo fin último es aportar al área de estudio de las alteraciones del lenguaje en EA una nueva evidencia sobre el proceso de deterioro del acceso léxico-semántico, se estructura en torno a los siguientes apartados: un marco teórico, que revisará las últimas investigaciones sobre las alteraciones del lenguaje en EA y, en particular sobre el déficit anómico; un marco metodológico, que explicará con detalle el estudio empírico llevado a cabo; los resultados obtenidos en el estudio de la anomia en personas mayores sin patología, personas con DCL y personas con EA; y una discusión final, que intentará ubicar los resultados obtenidos en el marco de la investigación actual sobre las alteraciones del lenguaje en la Enfermedad de Alzheimer y su valor predictivo en la detección temprana de esta demencia.

\section{Marco teórico}

La alteración del lenguaje es una de las manifestaciones más destacadas y tempranas de la Enfermedad de Alzheimer (Schröder, Wendelstein \& Felder, 2010). Las investigaciones más recientes han demostrado que el deterioro del lenguaje en EA es integral y afecta todos los niveles del sistema lingüístico y sus manifestaciones: la fonética segmental y suprasegmental (Mesulman, Rogalski, Wieneke, Hurley, Geula, Bigio, Thompson \& Weintraub, 2014; Martínez-Sánchez, Meilán, Vera-Ferrandiz, Carro, Pujante-Valverde, Ivanova \& Carcavilla, 2017); el nivel léxico y el nivel semántico (Szatloczki, Hoffmann, Vincze, Kálmán \& Pakaski, 2015; Lima Silagi et al., 2015); la gramática y la sintaxis (Ahmed, de Jager, Haigh \& Garrard, 2012; Ivanova, 2015); así como la construcción del discurso (Pistono, Jucla, Barbeau, Saint-Aubet, Lemesle, Calvet, Köpke, Puel \& Pariente, 2016). Dentro de este cuadro de afectación lingüística compleja, las alteraciones léxico-semánticas ocupan un lugar destacado, tanto por su mayor evidencialidad en la expresión verbal de los hablantes como por su papel central en la caracterización del lenguaje en EA. La representación más destacada de dichas alteraciones es la afasia anómica, que consiste en el deterioro multilateral del contenido y del acceso semántico (Reilly, Peelle, Antonucci \& Grossman, 2011), derivado del deterioro de la memoria como foco principal de EA y manifiesto primeramente en la dificultad de denominación.

Como proceso psicolingüístico, la denominación incluye tres subetapas: la conceptualización, que contiene la información acerca de un concepto (imagen mental, sus propiedades semánticas y funciones); el acceso léxico, que contiene la información acerca de un lema representativo del concepto; y la articulación, que contiene la información acerca de las propiedades de la codificación fonológica del concepto (Salehi, Mohsen \& Ghasisin, 2017). Se trata de un proceso semánticamente arbitrario y dependiente del contexto (Harvey \& Schnur, 2016), que se altera en subetapas distintas en diferentes condiciones de la vejez. Mientras que en hablantes 
mayores sin patologías los problemas de denominación y búsqueda de la palabra adecuada derivan de cambios en la subetapa de acceso léxico, en personas con demencia la alteración parece ubicarse en la subetapa conceptual (Salehi et al., 2017). Los procesos neurodegenerativos subyacentes a EA causan alteraciones en la organización y la estructura de los conocimientos semánticos, dando lugar a la pérdida o la degradación de los conceptos, sus atributos y enlaces entre ellos, y, en consecuencia, de las redes semánticas responsables de la activación de las representaciones léxicas y fonológicas (Willers, Feldman \& Allegri, 2008).

La interrupción de las redes semánticas en EA se manifiesta en la anomia, uno de los, si no el más, síntomas comunes manifiestos de la demencia (Papathanasiou, Coppens \& Potagas, 2013). A diferencia de otros síntomas de la afectación del lenguaje, la anomia en EA se detecta desde las fases iniciales de la demencia (Willers et al., 2008), manifestándose, en la producción espontánea, en pausas para buscar palabras (latencia) y, en la descripción de las imágenes, en la dificultad de nombrar objetos, contrastando con la preservación de la comprensión del significado léxico (Montagut, Sánchez-Valle, Castellví, Rami \& Molinuevo, 2010). En entrevistas clínicas, los hablantes con EA refieren que saben las respuestas a las preguntas que se le hacen, pero no las pueden formular (Edwards, 1993), mostrando afectación tanto del nivel propiamente léxico como del nivel conceptual.

Con el avance de la enfermedad, el hablante pierde la capacidad de reconocer el significado de las palabras (Ardila, 2005), 'olvidando' primero las palabras de baja frecuencia y luego, las de alta frecuencia de uso (Arboleda Ramírez, 2016). La evolución hacia EA, además, va asociada a la modulación de una variable de naturaleza semántica, la tipicidad, que se manifiesta en que los hablantes dementes producen mayor número de unidades léxicas típicas (Vita, Marra, Spinelli, Caprara, Scaricamazza, Castelli, Canulli, Gainotti \& Quaranta, 2014), es decir, aquellas que poseen un mayor número de rasgos característicos asociados a una categoría semántica (Riley \& Thompson, 2010).

Frente a la anomia propia del envejecimiento no patológico, la anomia debida a la demencia tipo Alzheimer interfiere de forma dramática en la comunicación del hablante, dando lugar a una discapacidad comunicativa funcional (Kempler \& Zalinski, 1994). En los casos de EA más castigados desde el punto de vista del lenguaje -aquellos que van acompañados por la variante logopénica de la afasia progresiva primaria - la anomia se presenta asociada a sustituciones fonológicas, parafasias semánticas, repetición anormal de palabras sueltas (Leyton, Hodges, Piguet \& Ballard, 2017) y la aparición de circunloquios, o frases sustitutorias para expresar el significado de la palabra que no se consigue recuperar (Cuetos, 2012). Las personas con EA acceden ante todo a la forma fonológica de la palabra y, en consecuencia, producen numerosas respuestas falsas a estímulos conceptuales (Moayedfar, Purmohammad, Shafa, Shafa \& Ghasisin, 2019). De estos, el acceso deteriorado 
afecta, ante todo, las entidades semánticamente únicas (como nombres propios o nombres de los lugares) frente a las entidades semánticamente genéricas (Montembeault, Brambati, Joubert, Boukadi, Chapleau, Laforce, Wilson, Macoir \& Rouleau, 2017), mientras que no parece haber deterioro discriminante entre el procesamiento de unidades léxicas concretas y abstractas (Joubert, Vallet, Montembeault, Boukadi, Wilson, Laforce, Rouleau \& Brambati, 2017).

Estudios recientes (Leyton et al., 2017) han revelado que la anomia de EA correlaciona primariamente con la degeneración del giro temporal superior izquierdo, área cortical responsable de numerosos procesos lingǘsticos (procesamiento fonológico, procesos gramaticales), entre los que destacan los procesos semánticos (Friederici, 2017). Una lesión o alteración en esta área cortical da lugar a la anomia pura, bajo cuyos efectos el hablante puede activar el significado que quiere transmitir, pero no puede acceder a las palabras para expresarlo (Cuetos, 2012). Estas dificultades de búsqueda y acceso léxico, que aparecen en las fases más tempranas de EA, provocan frecuentes interrupciones en la fluidez verbal de los hablantes; es decir, en su capacidad de producir un lenguaje espontáneo fluido sin pausas excesivas ni errores en la búsqueda y el uso de palabras (Butman, Allegri, Harris \& Drake, 2000). Al respecto, varios estudios recientes han venido afirmado que una correcta evaluación del déficit anómico podría ser clave para detectar el desarrollo de EA en sus estados más tempranos (Tsantali, Economidis \& Tsolaki, 2013). Para ello, se hace necesario diferenciar con rigor los problemas anómicos propios del inicio de la demencia de los problemas anómicos característicos del envejecimiento no patológico, al que nos referiremos bajo el término de senescencia no patológica (SNP), por un lado; y del deterioro cognitivo leve (DCL), un estado de deterioro cognitivo con altas probabilidades de evolución en EA (Gauthier, Reisberg, Zaudig, Petersen, Ritchie, Broich et al., 2006), por el otro. Diferenciar con precisión entre las alteraciones anómicas de estas tres condiciones de la vejez tiene, además del gran valor diagnóstico, una destacada importancia para la teoría lingüística: la comprensión de cómo va deteriorándose el acceso léxico-semántico con el declive cognitivo desde SNP hasta DCL y desde DCL hasta EA puede aportar datos significativos a la visión científica de la retrogénesis lingüística a lo largo de la vida.

Como modelo que explica la pérdida del lenguaje en EA, la teoría de la retrogénesis se sostiene en la idea de que el deterioro cognitivo propio de la demencia es un proceso proporcionalmente inverso a los patrones de adquisición ontogénica (Rubial-Álvarez, de Sola, Machado, Sintas, Böhm, Sánchez-Benavides, Langohr, Muñiz \& Peña-Casanova, 2013). Diferentes estudios han venido demostrando que la retrogénesis se basa en la alteración de mecanismos neuronales y biomoleculares (Stricker, Schweinsburg, Delano-Wood, Wierenga, Bangen, Haaland, Frank, Salmon \& Bondi, 2009) que, a su vez, se manifiesta en la pérdida de las habilidades funcionales (Reisberg, Franssen, Souren, Auer, Akram \& Kenowsky, 2002) y cognitivas, entre 
ellas, el habla y el lenguaje (Rogers \& Lasprilla, 2006). No obstante, en la literatura científica no hay consenso sobre si el lenguaje, como una de las funciones cognitivas más relevantes, está sujeto a los procesos retrogenerativos en su totalidad o si, por el contrario, se ven afectados únicamente algunos de sus niveles, por ejemplo, los más dependientes de la memoria. La evaluación del déficit anómico podría ser clave para confirmar si las habilidades lingüísticas basadas en los procedimientos mnésicos se someten o no a los principios de la retrogénesis.

Una de las mejoras técnicas para la evaluación de la producción verbal es la prueba de fluidez verbal (FV). En dicha prueba - un ejemplo de la misma podría ser el Set Test de Isaacs (Isaacs \& Akhtar, 1972)- el hablante tiene que producir el máximo número de palabras que pueda bajo un determinado tipo de estímulo semántico. El estímulo semántico da lugar a la prueba de fluidez verbal semántica (FVSem) y suele consistir en una categoría semántica concreta (animales, colores, muebles, etc.), sobre la que se pide producir tantas unidades léxicas cuantas se pueda: "Diga todas las palabras que pueda que pertenezcan a la categoría de animales". Otras tareas utilizan, en vez de un estímulo semántico, un estímulo fonológico que da lugar a la prueba de fluidez verbal fonológica (FVFon) y suele consistir en una pista fonémica: "Diga todas las palabras que pueda que empiecen por /p/". La utilidad de las pruebas de FV es notoria, en tanto que permite medir la facilidad de la producción verbal en términos de las funciones del lenguaje, aportando datos sobre el tamaño y la organización del vocabulario mental; la velocidad, las estrategias y los mecanismos de acceso léxicosemántico; la capacidad de denominación y la construcción del almacén semántico (Ramírez, Ostrosky-Solís, Fernández \& Ardila, 2005). En definitiva, se trata de una tarea que mide la actividad cognitiva compleja de la producción lingüística, la búsqueda mnésica y los procesos ejecutivos de acceso léxico (Rosser \& Hodges, 1994; Henry \& Crawford, 2004), que por su sencillez y utilidad es frecuentemente utilizada en la evaluación neuropsicológica de los trastornos del lenguaje.

En el estudio de la anomia en EA, la prueba de FV se ha utilizado con conveniente éxito. Se ha demostrado que la prueba de FVSem evoca mayor deterioro del lenguaje en hablantes dementes que la prueba de pista fonémica (Canning, Leach, Stuss, Ngo \& Black, 2004; Murphy, Rich \& Troyer, 2006); es decir, que el acceso a las redes semánticas y, por consiguiente, al almacén léxico, se encuentra más dañado que el procesamiento fonológico. Si bien algunas investigaciones (Cerella, 1985; Salthouse, 1996) han apuntado a que el déficit anómico en EA puede deberse al enlentecimiento cognitivo propio de la vejez, parece más razonable sostener que la retrogénesis cognitiva propia de EA deriva de manera directa en la alteración de redes léxicosemánticas de modo invertido (Simoes Loureiro \& Lefebvre, 2016).

De los resultados de varios estudios sobre la aplicabilidad de la prueba FVSem se conoce que la producción de unidades léxicas disminuye conforme transcurre el tiempo de la tarea -normalmente, de un minuto de duración- (Hurks, Vles, 
Hendriksen, Kalff, Feron, Kroes, van Zeben, Steyaert \& Jolles, 2006; Raboutet, Sauzéon, Corsini, Rodrigues, Langevin \& N'kaoua, 2010); y que las unidades léxicas de mayor frecuencia de uso se encuentran más disponibles y se activan de manera automática al principio de la prueba. La idea de la jerarquización del rendimiento en la prueba FVSem se basa en la observación de que la cantidad de unidades léxicas producidas por los hablantes desciende progresivamente, ya que implica diferentes procesos cognitivos subyacentes. Así, la producción de las primeras unidades léxicas por estímulo semántico se basa en la recuperación automatizada desde las redes semánticas, mientras que la producción sucesiva requiere un esfuerzo cognitivo superior debido a la saturación de las categorías semánticas y el acceso a unidades de menor frecuencia (Taporoski, Duarte, Pompéia, Sterr, Gómez, Alvim, Horimoto, Krieger, Vallada, Pereira, von Schantz \& Negrão, 2019). Con el paso del tiempo de la tarea, el repertorio léxico más disponible se satura, de forma que el acceso léxicosemántico pasa del modo automático al modo ejecutivo, requiriendo mayor esfuerzo cognitivo y presentando menor productividad (Hurks et al., 2006). Aunque la prueba FVSem mide ante todo los procesos de control ejecutivo sobre la memoria semántica (Shao, Janse, Visser \& Meyer, 2014), resulta apropiado distinguir dentro de la misma un subperiodo basado esencialmente en los procesos automatizados, de un subperiodo más dependiente de los procesos ejecutivos. Frente a fluencias verbales de otros tipos, como la fonológica, la fluidez semántica depende en mayor grado de funciones de recuperación semántica por clusters (Kraan, Stolwyk \& Testa, 2013), un proceso cognitivo automático (Raoux, Amieva, Le Goff, Auriacombe, Carcaillon, Letenneur \& Dartigues, 2008), así como de la integridad del almacén semántico (Henry, Crawford \& Phillips, 2004), que de los procesos ejecutivos. La función ejecutiva, como pudieron observar Whiteside, Kealey, Semla, Luu, Rice, Basso y Roper (2016), no es el único determinante de la fluidez verbal, ya que el propio procesamiento lingüístico desempeña un papel muy relevante en el rendimiento de producción verbal.

En vista de ello, resulta interesante averiguar cómo el estado cognitivo de un hablante mayor va reflejándose en la velocidad y la calidad de la fluidez verbal semántica como marcador de la retrogénesis lingüística. Es predecible que el análisis de la secuencia temporal de la fluidez verbal refleje procesos de acceso léxicosemántico afectados en diferente medida en hablantes sin deterioro cognitivo, hablantes con DCL y hablantes afectados por la demencia, sobre todo, atendiendo a que los procesos automatizados de acceso semántico son mejor preservados en EA (Arroyo-Anlló, Bellouard, Ingrand \& Gil, 2011).

\section{Marco metodológico}

Con el fin de verificar la afectación del acceso léxico-semántico con la evolución del deterioro cognitivo y averiguar las principales características de la alteración anómica en hablantes con demencia, realizamos un estudio empírico con personas 
mayores con senescencia no patológica (SNP), personas mayores con Deterioro Cognitivo Leve (DCL) y personas mayores con Enfermedad de Alzheimer (EA). El principal objetivo práctico del estudio consistió en analizar la secuencia en la cantidad de palabras generadas por los tres grupos de participantes en la tarea de FVSem con una modificación experimental: dividiendo el tiempo de duración de la prueba (un minuto) en cuatro intervalos de 15 segundos (Int1: 0-15 segundos; Int2: 15-30 segundos; Int3: 30-45 segundos; Int4: 45-60 segundos). La propuesta de la división del tiempo de duración de la prueba en cuatro intervalos se basó en la siguiente conjetura: si, como se acaba de mencionar supra, la denominación depende de procesos cognitivos distintos -son automáticos al principio pero dependen de la capacidad ejecutiva del hablante a posteriori-, el control temporal de la prueba de denominación permitirá evidenciar con mayor claridad el arranque del déficit anómico y, por tanto, diferenciar de forma más eficaz entre hablantes con y sin demencia.

La hipótesis subyacente a esta propuesta predice que, mientras que hablantes mayores sin patología (SNP) mostrarán una disminución paulatina en la evocación de palabras (producirán menos palabras conforme pasen los intervalos), hablantes mayores con déficit cognitivo mostrarán alteraciones anómicas con la siguiente distribución:

- personas con DCL ejecutarán igual que personas con SNP durante el primer intervalo (Int1: 0-15 segundos) pero mostrarán deterioro en los siguientes intervalos debido a la afectación del control ejecutivo de acceso léxicosemántico;

- personas con EA mostrarán deterioro en todos los intervalos debido a la afectación del acceso léxico-semántico automático y el deterioro más pronunciado del control ejecutivo de acceso léxico-semántico.

Estas hipótesis parten de los resultados de otros estudios sobre la fluidez verbal semántica en el deterioro cognitivo asociado a la vejez, que aplicaron la división de la prueba en intervalos de 15 segundos. En estas, personas con EA demostraron diferencias cuantitativas (número de unidades léxicas producidas) y cualitativas (intervalos de 15 segundos) frente a personas con DCL y personas mayores sin deterioro con valor clínico (Hall, Harvey, Vo \& O’Bryant, 2011). Algunos estudios sostuvieron que es la producción verbal durante el primer intervalo de 15 segundos la que se diferencia cualitativamente de otros intervalos en la prueba de fluidez semántica (Venegas \& Mansur, 2011).

\subsection{Participantes}

En este estudio participaron en total 209 personas mayores con diferentes condiciones cognitivas -SNP, DCL y EA- discriminadas principalmente entre sí gracias a la prueba Mini-Mental State Exploration, o MMSE (Folstein, Folstein \& 
McHugh, 1975). Todos los participantes dieron su consentimiento informado escrito para participar en el estudio, bien de forma personal bien por autorización de sus familiares tutores. La investigación se ajustó a los criterios éticos establecidos por el Comité de Bioética de la Universidad de Salamanca.

En la muestra, 126 participantes formaron el grupo de personas con senescencia no patológica (SNP). Su estado cognitivo no deteriorado fue confirmado a través de la evaluación mediante el test estandarizado y ponderado MMSE, en el que obtuvieron una media de 28,37 puntos (Desv.Est. = 1,9), superiores al valor de referencia para su edad. El grupo de personas con DCL fue formado por 48 personas. El deterioro cognitivo leve (DCL) se define como una alteración cognitiva (mayormente, de la memoria) no lo suficientemente grave (Lozano Gallego, Hernández Ferrándiz, Turró Garriga, Pericot Nierga, López-Pousa \& Vilatla Franch, 2009), que le permite a la persona afectada a mantener la independencia en sus habilidades funcionales y no repercute en su vida diaria. Los participantes con esta condición cumplieron los criterios del DCL establecidos por International Working Group on Mild Impairment (Winblad, Palmer, Kivipelto, Jelic, Fratiglioni et al., 2004) y obtuvieron una puntuación media de 22,90 (Desv.Est. = 4,35) en MMSE. La ausencia del deterioro en las actividades de la vida diaria, clave en la distinción entre DCL y demencia, fue confirmada mediante test Functional Activity Questionnaire (FAQ $<6)$. Todos los participantes con SNP y DCL fueron voluntarios de un centro de salud de atención primaria. Se confirmó que llevan una vida independiente. El grupo de personas con EA fue formado por 38 personas procedentes del Centro de Referencia Estatal de Atención a Personas con Enfermedad de Alzheimer y Otras Demencias (CRE Alzheimer). Su diagnóstico con EA fue confirmado por el servicio neurológico de CRE Alzheimer mediante el test MMSE, en el que obtuvieron una media de 18,29 puntos (Desv.Est $=5,77)-$ muy por debajo de la puntuación mínima de referencia para su edad-; así como mediante criterios NINCDS-ADRDA (Dubois, Feldman, Jacova, Dekosky, Barberger-Gateau \& Cummings, 2007) y la escala Global Deterioration Scale de Reisberg, que evidenció un deterioro cognitivo de leve a moderado (GDS 3 y 4).

Los tres grupos fueron contrastados para confirmar las diferencias significativas en el MMSE ponderado $\left(\mathrm{F}_{2,207}=132,692, \mathrm{p}<0.001\right)$. Los factores de exclusión de la muestra fueron: sospecha de la demencia de corte vascular; historia autoreportada de abuso de alcohol y/o drogas; déficits visuales o auditivos; y la presencia de síntomas depresivos (puntuación en la Escala de Goldberg > 6).

Las principales características de la muestra se recogen en la Tabla 1. 
Tabla 1. Muestra del estudio.

\begin{tabular}{|l|l|l|l|l|l|}
\hline \multirow{2}{*}{ Grupo } & Participantes & \multicolumn{2}{|c|}{ Edad media } & \multicolumn{2}{c|}{ Escolarización } \\
\cline { 2 - 6 } & Número total & Años & Desv.Est & Años & Desv.Est \\
\hline SNP & 126 & 75 & 7,5 & 9,41 & 3,83 \\
\hline DCL & 48 & 79 & 4,35 & 7,75 & 3,34 \\
\hline EA & 38 & 79 & 8,8 & 8,10 & 4,49 \\
\hline
\end{tabular}

\subsection{Materiales y procedimiento}

Todos los integrantes del estudio participaron en una prueba que incluyó, además del test MMSE (Winblad et al., 2004), la versión española del Set-Test de Isaacs (STI) (Isaacs \& Akhtar, 1972). STI es una prueba de fluidez verbal semántica, en la que se le pide al participante a producir en un minuto todos los nombres que pueda a partir de una categoría determinada: colores, animales, frutas y ciudades. Se considera una de las pruebas de evaluación más eficaces y adecuadas en el estudio del lenguaje en demencia, no solo por su brevedad y buena aceptación por los participantes, sino también por su gran valor en el análisis de la fluidez verbal, la denominación por categorías y la memoria semántica (Salazar \& Villar, 2007). La puntuación máxima de la prueba son 40 puntos, 10 puntos por cada categoría. El punto de corte para detectar una demencia es igual o menor a 27 en personas mayores. Además, se propone un punto de corte de 25 para considerar como probable la demencia tipo Alzheimer, obteniendo una sensibilidad del 87\% y una especificidad del 67\%, con un índice de mal clasificados equivalente a $24 \%$. Además de analizar los resultados generales del STI, evaluamos la cantidad de palabras generadas por los tres grupos según cuatro intervalos de tiempo de 15 segundos -Int1 (0-15 segundos), Int2 (15-30 segundos), Int3 (30-45 segundos) e Int4 (45-60 segundos) - para cada una de las categorías evaluadas. De esta forma, obtuvimos los datos descriptivos de todas las pruebas analizadas para cada grupo de participantes. Todos los datos obtenidos fueron analizados estadísticamente con el fin de determinar si existe una correspondencia entre el estado de deterioro cognitivo y la afectación léxico-semántica en personas mayores. Para ello, realizamos un test ANOVA de un factor (one-way ANOVA) separadamente para cada prueba, obteniendo su tamaño del efecto $\eta^{2}$ (Cohen, 1988). Como pruebas post-hoc, ejecutamos el test de comparación de medias y, además, realizamos un cálculo del tamaño del efecto utilizando la $d$ de Cohen (Cohen, 1988). Con el fin de evitar las diferencias debidas al nivel de ejecución en la comparación de la secuencia, obtuvimos la ratio de ejecución de cada participante en cada uno de los intervalos.

\section{Resultados}

Los resultados globales de nuestro estudio, que recogemos en la Tabla 2 , muestran que existen unas diferencias significativas y un tamaño de efecto muy alto entre los grupos de personas mayores con SNP, DCL y EA en la capacidad de acceso léxico- 
semántico $\left(\mathrm{F}_{2,206}=86.656, \mathrm{p}<0,001 ; \eta 2=0,46\right)$. Las personas con SNP muestran una capacidad de acceso léxico-semántico muy superior (media del SIT $=38$, Desv.Est. $=$ 4) a la de personas mayores con DCL (media del STI $=29$, Desv.Est. $=7$ ) y a la de personas mayores con EA (media de STI $=24$, Desv.Est. $=9$ ), siendo ambas tendencias estadísticamente significativas (SNP/DCL: $\mathrm{p}<0,001$, Cohen's $\mathrm{d}=1,42$; $\mathrm{r}=$ 0,60; SNP/EA: $\mathrm{p}<0,001 ; \mathrm{d}=1,89 ; \mathrm{r}=0,69)$. En este sentido, nuestro experimento replicó muy de cerca las puntuaciones obtenidas por los mismos grupos en la validación del STI en español $(\mathrm{ROC}=24,5$; sensibilidad $=98,4$; especificidad $=0.687$ ) (Pascual, Martínez, Modrego, Mostacero, López del Val \& Morales, 1990). Además, las personas con DCL ejecutaron la tarea significativamente mejor que los mayores con demencia $(\mathrm{p}<0,001 ; \mathrm{d}=0,62 ; \mathrm{r}=0,30)$. A nivel general de la prueba, pudimos observar diferencias significativas entre la ejecución de los grupos en Int1 $\left(\mathrm{F}_{2,206}=\right.$ 40.685; $\mathrm{p}<0,001 ; \eta 2=0,28)$, Int2 $\left(\mathrm{F}_{2,206}=20.555 ; \mathrm{p}<0,001 ; \eta 2=0,17\right)$ e Int4 (F, $\left.{ }_{206}=5.847 ; \mathrm{p}<0,01 ; \eta 2=0,05\right)$, que fueron particularmente manifiestas en Int1 e Int2 entre el grupo de SNP, por un lado, y los grupos de DCL y EA, por el otro $(p<0,01)$. El Gráfico 1 representa estos resultados de forma gráfica.

Tabla 2. Datos descriptivos y significación de contrastes entre grupos (SNP, DCL y EA) en la tarea de Fluidez Verbal Semántica (FVSem), en total y por intervalos.

\begin{tabular}{|c|c|c|c|}
\hline $\begin{array}{l}* \text { ANOVA, } \\
\text { significativo }\end{array}$ & $\begin{array}{c}\text { SNP } \\
\text { Media (Desv.Est.) }\end{array}$ & $\begin{array}{c}\text { DCL } \\
\text { Media (Desv.Est.) }\end{array}$ & $\begin{array}{c}\text { EA } \\
\text { Media (Desv.Est.) }\end{array}$ \\
\hline FVSemántica++ & $37.60(3.54)$ & $29.42(7.32) * *$ & $24.17(9.38) * * \dagger$ \\
\hline FVSem (Int1)++ & $6.40(1.65)$ & $4.60(1.61)^{* *}$ & $3.97(1.72)^{* *}$ \\
\hline FVSem (Int2)++ & $2.28(1.21)$ & $1.56(0.95)^{* *}$ & $1.04(0.82)^{* *}$ \\
\hline FVSem (Int3) & $0.65(0.68)$ & $0.78(0.62)$ & $0.79(0.69)$ \\
\hline FVSem (Int4) + & $0.22(0.37)$ & $0.45(0.51)$ & $0.41(0.45)$ \\
\hline FVColor++ & $9.13(1.39)$ & $7.48(2.14) * *$ & $6.26(2.45) * *+$ \\
\hline FV Color (Int1)++ & $6.29(2.19)$ & $4.90(1.93)^{* *}$ & $4.66(2.19)^{* *}$ \\
\hline FV Color (Int2) ++ & $2.06(1.78)$ & $1.52(1.24)$ & $0.51(0.78)^{* *} \dagger$ \\
\hline FV Color (Int3) & $0.62(0.89)$ & $0.79(1.01)$ & $0.80(1.60)$ \\
\hline FV Color (Int4) & $0.17(0.45)$ & $0.27(0.57)$ & $0.29(0.46)$ \\
\hline FV Animal++ & $\underline{9.67(1.60)}$ & $7.42(2.30) * *$ & $6.57(3.23) * *$ \\
\hline FV Animal (Int1)++ & $6.14(2.33)$ & $4.40(2.09)^{* *}$ & $3.66(1.92)^{* *}$ \\
\hline FV Animal (Int2) ++ & $2.51(2.10)$ & $1.65(1.52)^{*}$ & $1.31(1.28)^{*}$ \\
\hline FV Animal (Int3) & $0.80(1.16)$ & $0.79(1.01)$ & $0.97(1.17)$ \\
\hline FV Animal (Int4) & $0.21(0.64)$ & $0.58(0.96)^{*}$ & $0.63(0.88)^{*}$ \\
\hline FV Frutas ++ & $9.44(1.44)$ & $7.00(2.32) * *$ & $5.34(2.91) * * t$ \\
\hline FV Frutas (Int1)++ & $6.68(2.04)$ & $4.75(1.94)^{* *}$ & $3.66(2.54)^{* *}$ \\
\hline FV Frutas $(\operatorname{Int} 2)++$ & $1.87(1.47)$ & $1.13(1.21)^{*}$ & $0.91(1.20)^{* *}$ \\
\hline FV Frutas (Int3) & $0.55(091)$ & $0.71(0.90)$ & $0.57(0.81)$ \\
\hline FV Frutas (Int4) & $0.26(0.61)$ & $0.42(0.68)$ & $0.20(0.47)$ \\
\hline FV Ciudades ++ & $10.00(1.44)$ & $7.60(2.63) * *$ & $6.66(4.08) * *$ \\
\hline FV Ciudades (Int1)++ & $6.48(2.54)$ & $4.35(2.03)^{* *}$ & $3.91(2.42)^{* *}$ \\
\hline FV Ciudades (Int2)++ & $2.67(2.22)$ & $1.94(1.77)$ & $1.40(1.70)^{* *}$ \\
\hline FV Ciudades (Int3) & $0.66(1.32)$ & $0.83(1.08)$ & $0.83(1.74)$ \\
\hline FV Ciudades (Int4) & $0.28(0.83)$ & $0.52(0.85)$ & $0.51(0.95)$ \\
\hline
\end{tabular}

Leyenda: ${ }^{+}$Anova $(\mathrm{p}<0.05) ;{ }^{++}$Anova $(\mathrm{p}<0.01) ; *$ Significativamente peor que grupo control $(p<0.01) ; * *$ Significativamente peor que grupo control $(p<0.001)$; $†$ Significativamente peor que grupo DCL $(\mathrm{p}<0.01)$; † Significativamente peor que grupo DCL $(\mathrm{p}<0.001)$. 


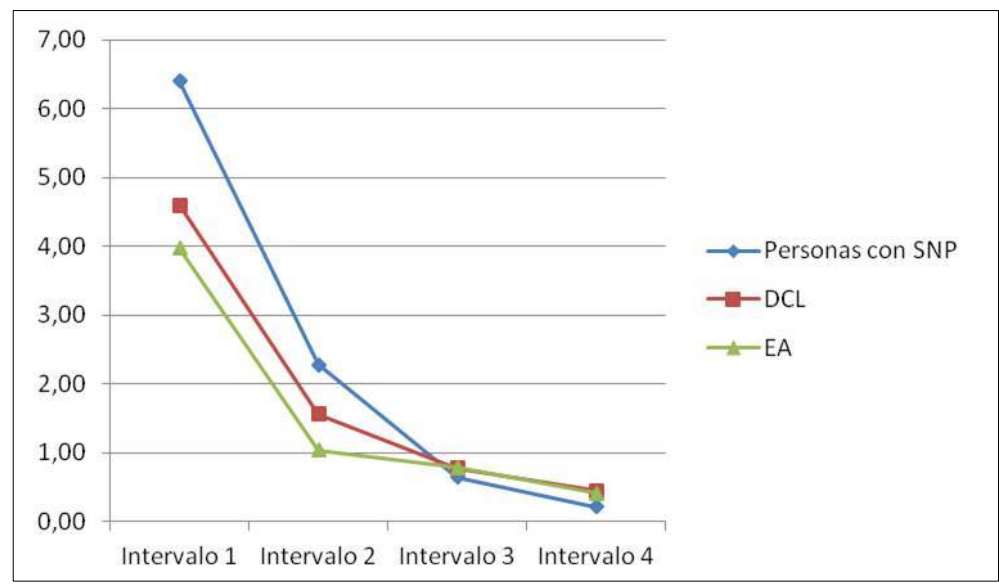

Gráfico 1. Número de palabras absolutas ejecutadas en la tarea de FV en cada intervalo de 15 segundos por cada grupo de mayores.

Para poder comparar la secuencia temporal de ejecución de la tarea de fluidez semántica de cada grupo experimental (SNP, DCL y EA) evitando los sesgos debidos a la diferencia en el número absoluto de palabras producidas por cada grupo, calculamos la ratio de ejecución de las unidades léxicas emitidas por cada grupo en cada uno de los cuatro intervalos de 15 segundos. De esta forma, pudimos evaluar la tasa de fluidez de cada participante sobre la base de su ejecución general. Para ello, realizamos la prueba ANOVA de medidas repetidas $3 \times 4$ con la variable tipo de muestra y la variable ratio por intervalo de tiempo. Con esta prueba pudimos encontrar diferencias entre los grupos en la ejecución de acceso léxico-semántico debidas a la variable ratio por intervalo de tiempo $\left(\mathrm{F}_{3,206}=723,908 ; \mathrm{p}<0,001 ; \eta 2=\right.$ 0,46). De forma importante, la diferencia entre los cuatro intervalos analizados fue significativa $(p<0,01)$ en las personas con $\mathrm{SNP}\left(\mathrm{F}_{3,204}=632.651 ; \mathrm{p}<0,001 ; \eta 2=\right.$ $0,90)$, personas con DCL $\left(\mathrm{F}_{3,204}=165.856 ; \mathrm{p}<0,001 ; \eta 2=0,71\right)$ y personas con $\mathrm{EA}$ $\left(F_{3,204}=104.965 ; \mathrm{p}<0,001 ; \eta 2=0.61\right)$. Además, encontramos efecto significativo de interacción entre el tipo de muestra (grupo de participantes) y la variable de ratio por intervalo $\left(\mathrm{F}_{6,206}=4.070 ; \mathrm{p}<0,01 ; \eta 2=0,07\right)$. En las comparaciones por pares de las secuencias temporales, tanto las personas con SNP como las personas con DCL mostraron diferencias entre los cuatro intervalos $(\mathrm{p}<0,01)$. Es decir, tanto las personas mayores con SNP como personas con deterioro cognitivo leve evidencian diferencias en su capacidad de acceso léxico-semántico con el avance temporal de la prueba. Las personas con EA, en cambio, mostraron diferencias entre Int1 y el resto de los intervalos $(\mathrm{p}<0,01)$, pero no entre Int2 e Int3. En cuanto a las comparaciones entre las diferentes muestras según el intervalo, en Int1 no encontramos diferencias en la ratio de palabras producidas por los tres grupos $\left(F_{2,206}=1.845\right)$. Sin embargo, sí observamos diferencias en Int2 $\left(F_{2,206}=8.260 ; \mathrm{p}<0,001 ; \eta 2=0.07\right)$, Int3 $\left(F_{2,206}=\right.$ 5.615; $\mathrm{p}<0,01 ; \eta 2=0,05)$ e Int4 $\left(\mathrm{F}_{2,206}=10.52 ; \mathrm{p}<0,001 ; \eta 2=0,09\right)($ Gráfico 2). 


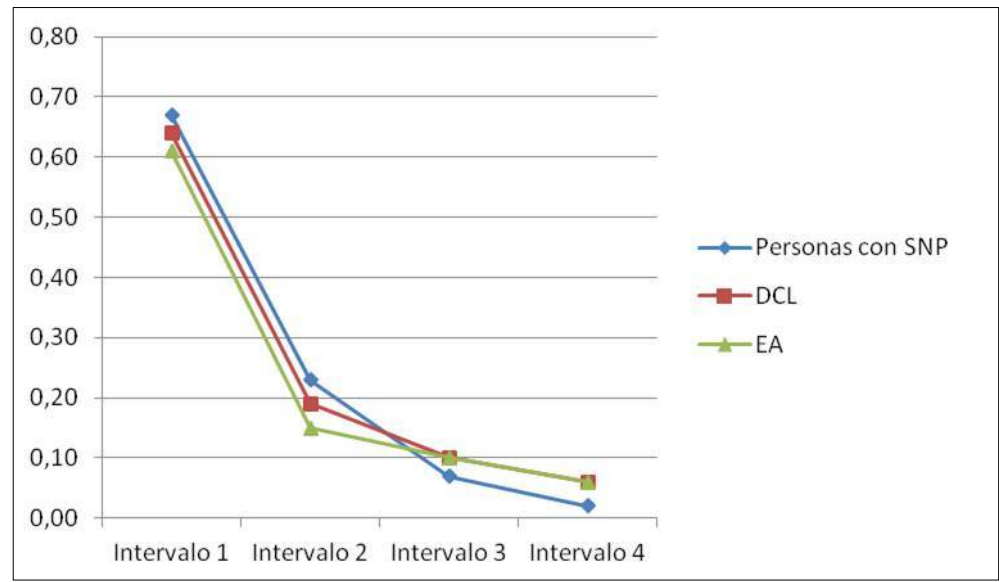

Gráfico 2. Ratio de ejecución de la tarea de FV en cada intervalo de 15 segundos.

Con el fin de comprobar las diferencias en el acceso a las redes semánticas según la categoría utilizada en la tarea de fluidez verbal (colores, animales, frutas y ciudades), comparamos las puntuaciones de las cuatro categorías utilizadas en un ANOVA mixto de medidas repetidas $4 \times 2$ con la variable tipo de muestra y la variable categoría de FVSem. Con esta prueba pudimos comprobar que hay diferencias en la ejecución léxico-semántica entre las cuatro categorías estudiadas $\left(\mathrm{F}_{3,618}=8.696 ; \mathrm{p}<0,01\right)$. Además, encontramos un efecto significativo de interacción entre la variable de tipo de muestra y las diferentes categorías $\left(\mathrm{F}_{3,618}=2.426 ; \mathrm{p}<0,05\right)$. Diferencias significativas y un alto tamaño del efecto entre grupos fueron encontrados en la tarea de FVSem de colores $\left(F_{2,206}=41.439 ; \mathrm{p}<0,001 ; \eta 2=0,29\right)$, FVSem de animales $\left(F_{2}\right.$, $\left.{ }_{206}=39.409 ; \mathrm{p}<0,001 ; \eta 2=0,28\right)$, FVSem de frutas $\left(F_{2,106}=86.656 ; \mathrm{p}<0,001 ; \eta 2=\right.$ $0,41)$ y $F V$ de ciudades $\left(F_{2,206}=33.819 ; \mathrm{p}<0,001 ; \eta 2=0,25\right)$. Si observamos las comparaciones (Figura 3), encontramos un rendimiento de acceso significativamente mejor en personas con SNP que en personas con DCL en todas las categorías: colores $(p<0,001 ; d=0,91 ; r=0,42) ;$ animales $(p<0,001 ; d=1,14 ; r=0,49)$; frutas $(p<0,001$; $\mathrm{d}=1,26 ; \mathrm{r}=0,53)$ y ciudades $(\mathrm{p}<0,001 ; \mathrm{d}=1,17 ; \mathrm{r}=0,50)$. El rendimiento de acceso significativamente mejor también fue encontrado para las cuatro categorías en las personas con SNP frente a las personas con EA: colores $(p<0,01 ; d=0,50 ; r=0,26)$; animales $(\mathrm{p}<0,001 ; \mathrm{d}=1,22 ; \mathrm{r}=0,52)$; frutas $(\mathrm{p}<0,001 ; \mathrm{d}=1,79 ; \mathrm{r}=0,67)$ y ciudades $(\mathrm{p}<$ $0,001 ; d=1,12 ; r=0,49)$. La diferencia entre las categorías analizadas fue hallada en la comparación entre las personas con DCL y personas con EA. Las personas con DCL ejecutaron mejor que las personas con EA en las categorías de colores $(p<0,01$; $\mathrm{d}=0,50 ; \mathrm{r}=0,26)$ y frutas $(\mathrm{p}<0,01 ; \mathrm{d}=0,63 ; \mathrm{r}=0,30)$, pero no encontramos diferencias entre estos dos grupos en las categorías de ciudades y animales. 


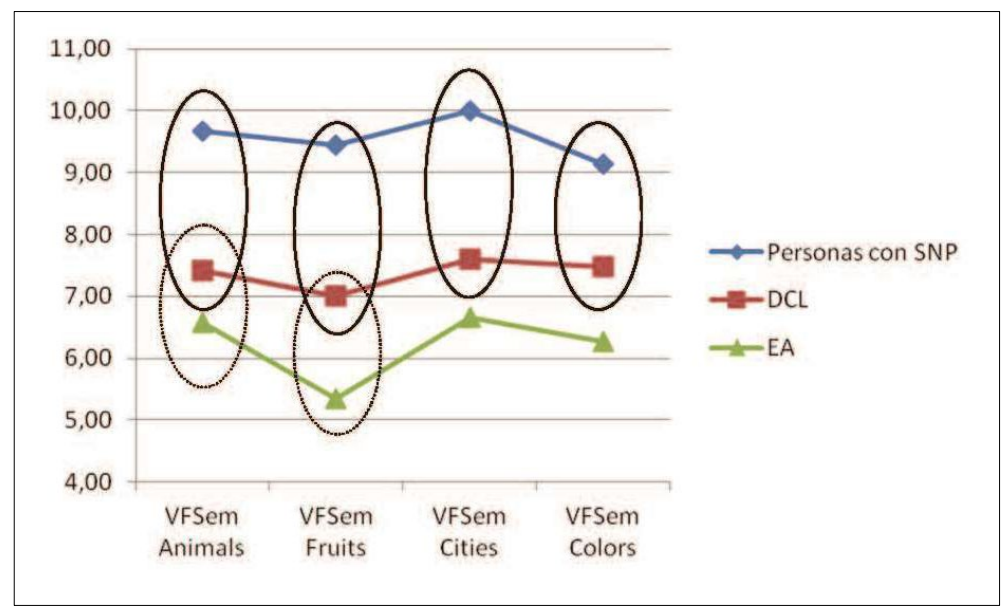

Gráfico 3. Ejecución en las diferentes tareas de Fluidez Verbal dependiendo del tipo de muestra.

\section{Discusión}

La anomia es una de las afectaciones lingüísticas más comunes en el envejecimiento patológico, que se manifiesta en el aumento progresivo de la dificultad de acceder a las redes léxico-semánticas conforme se intensifique el deterioro cognitivo de la persona mayor. Nuestro experimento basado en la tarea de FVSem nos permitió comprobar esta afectación en la muestra de hablantes con SNP, DCL y EA, y, además, confirmar que esta prueba tiene un alto potencial para distinguir cualitativa y cuantitativamente el grado de deterioro cognitivo en hablantes mayores, en línea con lo observado en otras investigaciones previas (Cuetos, Rodríguez-Ferreiro \& Menéndez, 2009).

La hipótesis del estudio, sin embargo, iba más allá y predecía que el grado de afectación del acceso léxico-semántico podría señalar con precisión el estado de deterioro cognitivo del hablante, permitiendo determinar si una persona con unos resultados concretos en la prueba de FVSem se encuentra en uno de los tres estadios analizados (SNP, DCL o EA). Nuestra hipótesis global se corroboró a nivel general: los tres grupos (SNP, DCL y EA) se diferencian significativamente en su capacidad de acceso léxico-semántico, confirmando que existe una retrogénesis lingüística paralela a la retrogénesis cognitiva propia del envejecimiento patológico. Es, por lo tanto, el primer paso en la validación de la idea de retrogénesis lingüística en EA, acorde a la manifestación de la retrogénesis cognitiva definida por Rubial-Álvarez et al. (2013) como un declive progresivo inverso en las habilidades funcionales y cognitivas de las personas con demencia. Era predecible, de acuerdo con lo que se ha mencionado en el Marco Teórico, que la retrogénesis afectaría ante todo los niveles más dependientes de la memoria, como es la habilidad léxico-semántica. Esta observación concuerda con trabajos previos, que concluyeron sobre la curva en forma de $U$ de la involución 
semántica en EA en comparación con el desarrollo semántico en niños (Simoes Loureiro \& Lefebvre, 2016). No obstante, en lo que atañe a la hipótesis específica, que predecía la dependencia de la capacidad de acceso léxico-semántico del intervalo temporal de la prueba y, por tanto, de diferentes procesos cognitivos (acceso léxicosemántico automático vs. acceso léxico-semántico por control ejecutivo), solo pudimos observar su corroboración entre los grupos SNP y DCL, pero no en EA.

En los grupos SNP y DCL, la secuencia de decaimiento de la ejecución del acceso léxico-semántico es significativa a lo largo de los 4 intervalos (Int1-Int4) y se muestra paralela en ambos grupos. En otras palabras, las personas mayores con SNP y DCL exhiben un descenso continuo significativo de su capacidad de acceso léxicosemántico con el avance de los intervalos, tanto en la ejecución absoluta de la prueba ( $\mathrm{n}^{\mathrm{o}}$ total de unidades léxicas) como en la ratio relativa del grupo. Sin embargo, las personas con EA -contrariamente a lo que esperábamos- mostraron únicamente diferencias significativas entre Int1 y el resto de los intervalos, pero no entre intervalos a partir del Int2. Existe, por lo tanto, una clara diferencia en la capacidad del acceso léxico-semántico automático y controlado en hablantes con demencia, manifestando una alteración global de toda la capacidad léxico-semántica dependiente de los procesos ejecutivos.

Resultados semejantes fueron obtenidos en otros estudios (Hall et al., 2011) que demostraron que las personas con demencia producen un número significativamente más bajo de unidades léxicas a partir del Int2. Una de las posibles explicaciones de esta observación podría apoyarse en la idea que hemos sostenido arriba acerca de la mayor implicación de los procesos automáticos al principio de la prueba de fluidez semántica y de los procesos ejecutivos a su final: tal y como corroboraron Weakley y SchmitterEdgecombe (2014), personas con EA muestran un peor rendimiento en acceso léxicosemántico en intervalos sucesivos al primero debido al deterioro de habilidades ejecutivas relacionadas con la búsqueda y recuperación léxico-semántica. La constancia del empeoramiento significativo a partir del primer intervalo también fue observada en pruebas con divisiones de la duración estandarizada de la prueba de 60 segundos en intervalos distintos. Así, por ejemplo, la división del minuto en 3 intervalos de 20 segundos permitió concluir a Demetriou y Holtzer (2017) que la diferencia significativa observada entre la producción durante el primer intervalo y la producción de los intervalos sucesivos se debe a que la fluidez verbal se basa en dos procesos distintos: se trata, en las etapas iniciales, de procesos de recuperación semi-automática desde el topicón (una especie de almacén mnésico a largo plazo que incluye unidades léxicas ordinarias), y, en las etapas sucesivas, de procesos de recuperación forzosa desde almacenes más extensos y menos disponibles. Desde el punto de vista metodológico, es una observación importante, puesto que aboga por la necesidad de ejecutar las pruebas de FVSem con un intervalo mínimo de un minuto -y no de medio 
minuto, como ocurre en muchas pruebas de cribado- para que la discriminación entre personas con DCL y personas con EA sea fiable.

Mientras tanto, los hablantes sin patología y los hablantes con el deterioro cognitivo no demencial evidencian no una caída brusca en la fluidez verbal semántica, sino una secuenciación continua en la disminución de la ratio de unidades léxicas evocadas. Ello apunta, con toda certeza, a una mejor preservación de las habilidades de acceso léxico-semántico automático y controlado en estados cognitivos no patológicos, si bien el progreso de SNP a DCL influye considerablemente en la ratio de unidades léxicas accedidas. Asimismo, nuestros resultados apuntan a que, en general, la preservación de los procesos automáticos de acceso léxico-semántico es mayor y mejor que el mantenimiento de los procesos de acceso léxico-semántico por ejecución controlada en la vejez. En este sentido, nuestra investigación replica los resultados de otros estudios anteriores (Duong, Whitehead, Hanratty \& Chertkow, 2006), que sostienen el deterioro anterior del acceso semántico controlado frente al acceso automático, preservado durante más tiempo en las personas mayores.

En cuanto a la caracterización del acceso léxico-semántico y de la anomia como rasgo diferenciador temprano de EA, con nuestro estudio pudimos corroborar que la alteración de esta función en la demencia tiene que ver con la afectación de las redes semánticas. Los resultados obtenidos para diferentes categorías (animales, colores, frutas y ciudades) nos permitieron ver que funcionan de manera distinta en la diferenciación significativa entre los hablantes con DCL y los hablantes con EA colores y frutas son dos categorías que permiten discriminar entre los dos grupos- y, por lo tanto, abogan porque en la demencia las alteraciones anómicas dependen del deterioro de redes semánticas específicas. Esta observación es importante desde el punto de vista metodológico, puesto que en muchos cribados se evalúan otras categorías semánticas -por ejemplo, animales- que, según nuestro estudio, no son sensibles a las diferencias anómicas entre el deterioro cognitivo no patológico y la demencia.

Los resultados de nuestra investigación tienen implicaciones importantes para la teoría lingüística, en particular, para la comprensión de la dependencia de las habilidades lingüísticas específicas del ser humano de su estado cognitivo. En la investigación más reciente sobre el lenguaje en EA, se ha venido sosteniendo la hipótesis sobre la involución lingüística asociada a la demencia que, al menos a nivel léxico-semántico, toma forma de retrogénesis, que replica los estadíos de adquisición del lenguaje a la inversa (Simoes Loureiro \& Lefebvre, 2016). La denominación en concreto da lugar a la retrogénesis nominal, que se manifiesta primeramente en la pérdida de conceptos y unidades léxicas adquiridas de forma tardía (Kim, Yoon, Lee, Baek, Sohn \& Na, 2011). Parece importante añadir que, además de la edad de adquisición del léxico - una variable con incidencia predecible en el mantenimiento de redes semánticas- la composición del repertorio léxico-semántico accesible depende 
sustancialmente de la frecuencia de uso de las unidades que lo compongan. En nuestro estudio, las categorías de colores y frutas cumplen con ambos criterios frente a las categorías de animales y ciudades, que, además, se caracterizan por formar series más abiertas y más subjetivamente compuestas.

\section{CONCLUSIONES}

Con este estudio pudimos comprobar que la habilidad léxico-semántica de las personas con la Enfermedad de Alzheimer se deteriora desde el inicio de la demencia de una forma específica, que la permite detectar de modo fehaciente a través de la prueba de Fluidez Verbal Semántica. Detrás de la alteración de esta capacidad lingüística se encuentra el deterioro del acceso controlado a las redes semánticas que, como bien es sabido de la litertura científica, se postula como un síntoma temprano de la enfermedad debido a cambios degenerativos en la memoria.

Las alteraciones en el acceso léxico-semántico propias de EA se manifiestan a partir del segundo cuarto del minuto (15 segundos en adelante) de la prueba de FVSem, sugiriendo que el formato más adecuado para su aplicación no debe ser inferior a 60 segundos. Dos categorías semánticas son clave para poder diferenciar a personas con EA de personas con DCL por problemas anómicos -colores y frutas-, que se definen por mayor homogeneidad composicional intersujeto y mayor diversidad composicional en lo que se refiere a la edad de adquisición y frecuencia de uso. A partir de los datos obtenidos, sería conveniente estudiar la diversidad y la calidad de las unidades léxicas que componen dichas categorías semánticas, con el fin de determinar cómo el deterioro cognitivo repercute en la riqueza léxico-semántica de los hablantes afectados por la demencia.

\section{REFERENCIAS BIBLIOGRÁFICAS}

Ahmed, S., de Jager, C. A., Haigh, A. M. \& Garrard, P. (2012). Logopenic aphasia in Alzheimer's disease: Clinical variant or clinical feature? Journal of Neurology, Neurosurgery, and Psychiatry, 83(11), 1056-1062.

Arboleda Ramírez, A. (2016). Diagnóstico neuropsicológico de la anomia: Algo más que una prueba paramétrica. Acta Neurológica Colombiana, 32(4), 273-274.

Ardila, A. (2005). Las afasias. Jalisco: Universidad de Guadalajara.

Arroyo-Anlló, E. V., Bellouard, S., Ingrand, P. \& Gil, R. (2011). Effects of automatic/controlled access processes on semantic memory in Alzheimer's disease. Journal of Alzheimer's Disease, 25(3), 525-533.

Becker, J. T. \& Overman, A. A. (2002). El déficit de la memoria semántica en la enfermedad de Alzheimer. Revista de Neurología, 35(08), 777-783. 
Butman, J., Allegri, R. F., Harris, P. \& Drake, M. (2000). Fluencia verbal en español. Datos normativos en Argentina. Medicina, 60(5/1), 561-564.

Canning, S. J., Leach, L., Stuss, D. T., Ngo, L. \& Black, S. E. (2004). Diagnostic utility of abbreviated fluency measures in Alzheimer`s disease and vascular dementia. Neurology, 62, 556-562.

Cerella, J. (1985). Information processing rates in the elderly. Psychological Bulletin, 98, 67-83.

Cohen, J. (1988). Statistical power analysis for the behavioral sciences (2nd edition). Hillsdate: LEA.

Cuetos, F. (2003). Anomia: La dificultad para recordar palabras. Madrid: TEA.

Cuetos, F. (2012). The role of aphasic disorders in the study of the brain-language relationship. En C. Boeckx, M. C. Horno-Chéliz \& J. L. Mendívil-Giró (Eds.), Language, from a Biological Point of View. Current Issues in Biolinguistics (pp. 143164). Cambridge: Cambridge Scholars.

Cuetos, F., Rodríguez-Ferreiro, J. \& Menéndez, M. (2009). Semantic markers in the diagnosis of neurodegenerative dementias. Dementia and Geriatric Cognitive Disorders, 28(3), 267-274.

Demetriou, E. \& Holtzer, R. (2017). Mild cognitive impairment moderate the effect of time on verbal fluency performance. Journal of the International Neuropsychological Society, 23(1), 44-55.

Dubois, B., Feldman, H. H., Jacova, C., Dekosky, S. T., Barberger-Gateau, P. \& Cummings J. (2007). Research criteria for the diagnosis of Alzheimer's disease: revising the NINCDS-ADRDA criteria. Lancet Neurology, 6, 734-746.

Duong, A., Whitehead, V., Hanratty, K. \& Chertkow, H. (2006). The nature of lexicosemantic processing deficits in mild cognitive impairment. Neuropsychologia, 44(10), 1928-1935.

Edwards, A. J. (1993). Dementia. Nueva York: Springer.

Folstein, M. F., Folstein, S. E. \& McHugh, P. E. (1975). Mini mental state: A practical method for grading the cognitive state of patients for the clinician. Journal of Psychiatric Research, 12, 189-198.

Friederici, A. D. (2017). Language in our brain. The origins of a uniquely buman capacity. Cambridge: The MIT Press.

Gauthier, S., Reisberg, B., Zaudig, M., Petersen, R. C., Ritchie, K., Broich, K., ... \& Cummings, J. L. (2006). Mild cognitive impairment. Lancet, 15, 1262-1270. 
Hall, J. R., Harvey, M., Vo, H. T. \& O’Bryant, S. E. (2011). Performance on a measure of category fluency in cognitively impaired elderly. Aging, Neuropsychology, and Cognition, 18(3), 353-361.

Harvey, D. Y. \& Schnur, T. (2016). Different loci of semantic interference in picture naming vs. word-picture matching task. Frontiers in Psychology: Accessing conceptual representations for speaking, 7, 31-49.

Henry, J. D. \& Crawford, J. R. (2004). A meta-analytic review of verbal fluency performance following focal cortical lesions. Neuropsychology, 18, 284-295.

Henry, J. D., Crawford, J. R. \& Phillips, L. H. (2004). Verbal fluency performance in dementia of the Alzheimer's type: a meta-analysis. Neuropsychologia, 42(9), 1212-1222.

Hurks, P. P., Vles, J. S., Hendriksen, J. G., Kalff, A. C., Feron, F .J., Kroes, M., van Zeben, T. M., Steyaert, J. \& Jolles, J. (2006). Semantic category fluency versus initial letter fluency over 60 seconds as a measure of automatic and controlled processing in healthy school-aged children. Journal of Clinical \& Experimenal Neuropsychology, 28, 684-695.

Isaacs, B. \& Akhtar, A. J. (1972). The set test: A rapid test of mental function in old people. Age Ageing, 1(4), 222-226.

Ivanova, O. (2015). Diseño del prototipo de la prueba normativa para la evaluación pre-clínica del trastorno semántico-sintáctico en la Enfermedad de Alzheimer. Trabajo de Fin de Máster inédito. Salamanca: USAL.

Jódar Vicente, M. (Coord.), Barroso Ribal, J., Brun i Gasca, C., Dorado Mesa, M., García Jiménez, A., Martín Plasencia, P. \& Nieto Barco, A. (2005). Trastornos del lenguaje y la memoria. Barcelona: Editorial UOC.

Joubert, S., Vallet, G. T., Montembeault, M., Boukadi, M., Wilson, M. A., Laforce, R. J., Rouleau, I. \& Brambati, S. M. (2017). Comprehension of concrete and abstract words in semantic variant of primary progressive aphasia and Alzheimer's disease: A behavioural and neuroimaging study. Brain and Language, 170, 93-102.

Kim, H., Yoon, J. H., Lee, J. E., Baek, E. J., Sohn, Y. H. \& Na, D. L. (2011). Is confrontation naming performance in Alzheimer's disease the nominal linguistic retrogenesis of normal development? European Neurology, 66(4), 195199.

Kempler, D. \& Zelinski, E. M. (1994). Language in dementia and normal aging. En F. A. Huppert, C. Brayne \& D. W. O’Connor (Eds.), Dementia and normal aging (pp. 331-365). Cambridge: Cambridge University Press. 
Kraan, C., Stolwyk, R. J. \& Testa, R. (2013). The abilities associated with verbal fluency performance in a young, healthy population are multifactorial and differ across fluency variants. Applied Neuropsychology: Adult, 20, 159-168.

Laine, M. \& Martin, N. (2006). Anomia: Theoretical and clinical aspects. Hove: Psychology Press.

Leyton, C. E., Hodges, J. R., Piguet, O. \& Ballard, K. J. (2017). Common and divergent neural correlates of anomia in amnesic and logopenic presentations of Alzheimer's disease. Cortex, 86, 45-54.

Lima Silagi, M., Ferreira Bertolucci, P. H. \& Zazo Ortiz, K. (2015). Naming ability in patients with mild to moderate Alzheimer's disease: What changes occur with the evolution of the disease? Clinics, 70(6), 423-428.

Lozano Gallego, M., Hernández Ferrándiz, M., Turró Garriga, O., Pericot Nierga, I., López-Pousa, S. \& Vilatla Franch, J. (2009). Validación del Montreal Cognitive Assessmnet (MoCA): Test de cribado para el deterioro cognitivo leve. Datos preliminares. Alžbeimer. Realidades e investigación en demencia, 43, 4-11.

Martínez-Sánchez, F., Meilán, J. J. G., Vera-Ferrandiz, J. A., Carro, J., PujanteValverde, I., Ivanova, O. \& Carcavilla, N. (2017). Speech rhythm alterations in Spanish-speaking individuals with Alzheimer's disease. Aging, Neuropsychology and Cognition, 24(4), 418-434.

Mesulman, M., Rogalski, E., Wieneke, C., Hurley, R. S., Geula, C., Bigio, E., Thompson, C. \& Weintraub, S. (2014). Primary progressive aphasia and the evolving neurology of the language network. Nature Reviews Neurology, 10(10), 554-569.

Moayedfar, S., Purmohammad, S., Shafa, N., Shafa, N. \& Ghasisin, L. (2019). Analysis of naming processing stages in patients with mild Alzheimer. Applied Neuropsychology: Adult [en línea].

Montagut, N., Sánchez-Valle, R., Castellví, M., Rami, L. \& Molinuevo, J. L. (2010). Reaprendizaje de vocabulario. Análisis comparativo entre un caso de demencia semántica y enfermedad de Alzheimer con afectación predominante del lenguaje. Revista de Neurología, 50(3), 152-156.

Montembeault, M., Brambati, S. M., Joubert, S., Boukadi, M., Chapleau, M., Laforce, R. J., Wilson, M. A., Macoir, J. \& Rouleau, I. (2017). Naming unique entities in the semantic variant of primary progressive aphasia and Alzheimer's disease: Towards a better understanding of the semantic impairment. Neuropsychologia, 95, 11-20. 
Murphy, K. J., Rich, J. B. \& Troyer, A. K. (2006). Verbal fluency patterns in amnestic mild cognitive impairment are characteristic of Alzheimer's type dementia. Journal of the International Neuropsychological Society, 12, 570-574.

Palacio, C. A. \& Toro, A. C. (2018). Psiquiatría. Medellín: CIB.

Papathanasiou, I., Coppens, P. \& Potagas, C. (2013). Aphasia and related Neurogenic Communication Disorders. Burlington: Jones \& Bartlett Learning.

Pascual, L. F., Martínez, J. V., Modrego, P., Mostacero, E., López del Val, J. \& Morales, F. (1990). El set-test en el diagnóstico de la demencia. Neurología, 5, 82-85.

Pistono, A., Jucla, M., Barbeau, E., Saint-Aubet, L., Lemesle, B., Calvet, B., Köpke, B., Puel, M. \& Pariente, J. (2016). Pauses during autobiographical discourse reflect episodic memory processes in early Alzheimer's diseas. Journal of Alzheimer's disease, 50(3), 687-698.

Raboutet, C., Sauzéon, H., Corsini, M. M., Rodrigues, J., Langevin, S. \& N’Kaoua, B. (2010). Performance on a semantic verbal fluency task across time: Dissociation between clustering, switching and categorical exploitation processes. Journal of Clinical and Experimental Neuropsychology, 32, 268-280.

Ramírez, M., Ostrosky-Solís, F., Fernández, A. \& Ardila, A. (2005). Fluidez verbal semántica en hispanohablantes: Un análisis comparativo. Revista de Neurología, 41(8), 463-468.

Raoux, N., Amieva, H., Le Goff, M., Auriacombe, S., Carcaillon, L., Letenneur, L. \& Dartigues, J. F. (2008). Clustering and switching processes in semantic verbal fluency in the course of Alzheimer's disease subjects: results from the PAQUID longitudinal study. Cortex, 44(9), 1188-1196.

Reilly, J., Peelle, J., Antonucci, S. \& Grossman, M. (2011). Anomia as a marker of distinct semantic memory impairments in Alzheimer's disease and semantic dementia. Neuropsychology, 25(4), 413-426.

Reisberg, B., Franssen, E. H., Souren, L. E., Auer, S. R., Akram, I. \& Kenowsky, S. (2002). Evidence and mechanisms of retrogenesis in Alzheimer's and other dementias: Management and treatment import. American Journal of Alzheimer's Disease \& Other Dementias, 17(4), 202-212.

Riley, E. A. \& Thompson, C. K. (2010). Semantic typicality effects in acquired dyslexia: Evidence for semantic impairment in deep dyslexia. Aphasiology, 24(6-8), 802-813. 
Rogers, H. \& Lasprilla, J. C. A. (2006). Retrogenesis theory in Alzheimer's disease: Evidence and clinical implications. Anales de psicología, 22(2), 260-266.

Rosser, A. \& Hodges, J. R. (1994). Initial letter and semantic category fluency in Alzheimer's disease, Huntington's disease, and progressive supranuclear palsy. Journal of Neurology, Neurosurgery, and Psychiatry, 57(11), 1389-1394.

Rubial-Álvarez, S., de Sola, S., Machado, M. C., Sintas, E., Böhm, P., SánchezBenavides, G., Langohr, K., Muñiz, R. \& Peña-Casanova, J. (2013). The comparison of cognitive and functional performance in children and Alzheimer's disease supports the retrogenesis model. Journal of Alzheimer's Disease, 33(1), 191-203.

Salazar, C. \& Villar, S. (2007). Evaluación neuropsicológica e intervención en demencias: La Enfermedad de Alzheimer. Granada: CAAP Formación.

Salehi, M., Mohsen, R. \& Ghasisin, L. (2017). Lexical retrieval or semantic knowledge? Which one causes naming errors in patients with mild and moderate Alzheimer's Disease? Dementia and Geriatric Cognitive Disorders Extra, 7(3), 419429.

Salthouse, T. A. (1996). The processing-speed theory of adult age differences in cognition. Psychological Review, 103, 403-428.

Schröder, J., Wendelstein, B. \& Felder, E. (2010). Language in the preclinical stage of Alzheimer's Disease. Content and complexity in biographic interviews of the ILSE study. Klinische Neuropsysiologie, 41, 360.

Shao, Z., Janse, E., Visser, K. \& Meyer, A. S. (2014). What do verbal fluency tasks measure? Predictors of verbal fluency performance in older adults. Frontiers in Psychology, 5, 772.

Simoes Loureiro, I. \& Lefebvre, L. (2016). Retrogenesis of semantic knowledge: Comparative approach of acquisition and deterioration of concepts in semantic memory. Neuropsychology, 30(7), 853-859.

Stricker, N. H., Schweinsburg, B. C., Delano-Wood, L., Wierenga, C. E., Bangen, K. J., Haaland, K. Y., Frank, L. R., Salmon, D. P. \& Bondi, M. (2009). Decreased white matter integrity in late-myelinating fiber pathways in Alzheimer's disease supports retrogenesis. NeuroImage, 45(1), 10-16.

Szatloczki G., Hoffmann I., Vincze V., Kálmán, J. \& Pakaski, M. (2015). Speaking in Alzheimer's disease, is that an early sign? Importance of changes in language abilities in Alzheimer's disease. Frontiers in Aging Neuroscience, 20(7), 195. 
Taporoski, T. P., Duarte, N. E., Pompéia, S., Sterr, A., Gómez, L. M., Alvim, R. O., Horimoto, A. R. V. R., Krieger, J. E., Vallada, H., Pereira, A. C., von Schantz, M. \& Negrão, A. B. (2019). Heritability of semantic fluency task using timeinterval analysis. PloS One, 14(6), e0217814.

Tsantali, E., Economidis, D. \& Tsolaki, M. (2013). Could language deficits really differentiate Mild Cognitive Impairment (MCI) from mild Alzheimer's disease? Archives of Gerontology and Geriatrics, 57, 263-270.

Venegas, M. J. \& Mansur, L. L. (2011). Verbal fluency: Effect of time on item generation. Dementia \& Neuropsychologia, 5(2), 104-107.

Viloria Jiménez, A., Gil Gregorio, P. \& Yubero Pancorbo, R. (2009). ¿'Toda pérdida de memoria es alzhéimer? Nuevas miradas sobre el envejecimiento (pp. 157-188). Madrid: IMSERSO.

Vita, M. G., Marra, C., Spinelli, P., Caprara, A., Scaricamazza, E., Castelli, D., Canulli, S., Gainotti, G. \& Quaranta, D. (2014). Typicality of words produced on a semantic fluency task in amnesic mild cognitive impairment: Linguistic analysis and risk of conversion to dementia. Journal of Alzheimer's Disease, 42(4), 1171-1178.

Weakley, A. \& Schmitter-Edgecombe, M. (2014). Analysis of verbal fluency ability in Alzheimer's disease: The role of clustering, switching and semantic proximities. Archives of Clinical Neuropsychology, 29(3), 256-268.

Whiteside, D. M., Kealey, T., Semla, M., Luu, H., Rice, L., Basso, M. R. \& Roper, B. (2016). Verbal fluency: Language or executive function measure? Applied Neuropsychology: Adult, 23(1), 29-34.

Willers, I. F., Feldman, M. L. \& Allegri, R. F. (2008). Subclinical naming errors in mild cognitive impairment. A semantic deficit? Dementia \& Nueropsychologia, 2(3), 217-222.

Winblad, B., Palmer, K., Kivipelto, M., Jelic, V., Fratiglioni, L., Wahlund, L.O,... \& Arai, H. (2004). Mild cognitive impairment-beyond controversies, towards a consensus: report of the International Working Group on Mild Cognitive Impairment. Journal of Internal Medicine, 256, 240-246. 